\title{
TENSOR PRODUCTS OF FUNCTION SPACES
}

\author{
BY MARIO MILMAN
}

Communicated by Alberto P. Calderón, April 8, 1976

1. In this note we give necessary and sufficient conditions for containments $X\left(\Omega_{1}\right) \hat{\otimes}_{\pi} Y\left(\Omega_{2}\right) \subseteq Z\left(\Omega_{1} \times \Omega_{2}\right)$ to hold for a large class of function spaces (cf. [4]). We also study tensor products of rearrangement invariant spaces (r.i.s.) as $L^{1}$ (resp. $\left.L^{\infty}\right)$ modules.

The justification for considering this problem is given by the fact that using duality we obtain continuity results for integral operators, convolution operators and diagonal operators.

Our results extend and clarify previous work by O'Neil [7], Torchinsky [10], and the author [5], [6].

2. Let $X(0, \infty)$ be a r.i. space, with fundamental function $\phi_{X}$ we let

$$
\Lambda\left(\phi_{X}, C\right)=\left\{f \in M(0, \infty): \int C\left(f^{* *}(t) \phi_{X}(t)\right) \frac{d t}{t}<\infty\right\}
$$

where $C$ is a Young's function. These spaces appear naturally in interpolation theory (cf. [1], [10]) and generalize the Lorentz $L(p, q)$ spaces as well as the Orlicz spaces. Their use allows us to unify some aspects of the theory for these classes of spaces.

3. Let $X(0, \infty), Y(0, \infty), Z[(0, \infty) \times(0, \infty)]$ be r.i. spaces. We denote by $\hat{Z}(0, \infty)$ the Luxemburg representation of $Z$ (cf. [4]).

THEOREM 1. The following conditions are necessary for $X \hat{\otimes}_{\pi} Y \subseteq Z$ to hold,

(i) $X \subseteq \hat{Z}, Y \subseteq \hat{Z}$;

(ii) $\exists \theta>0$ such that

$$
\phi_{Z}(t \cdot s) \leqslant \theta \phi_{X}(t) \phi_{Y}(s) \quad \forall t, s>0 .
$$

THEOREM 2. Let $X$ be a Lorentz $\Lambda_{\phi_{X}}$ space; then a necessary and sufficient condition for $X \hat{\otimes}_{\pi} Y \subseteq Z$ to hold is that $\exists \ni>0$ such that $\left\|E_{1 / s}\right\|_{Y \rightarrow \hat{Z}} \leqslant$ $\theta \phi_{X}(s) \forall s>0$, where $\left(E_{1 / s} f\right)(t)=f(t / s)$.

Our next result gives an estimate of the maximal rearrangement of a tensor product operator.

\section{THEOREM $3 .^{1}$ Let $T$ be a bilinear operator such that}

AMS (MOS) subject classifications (1970). Primary 46E30, 46E35, 46M05.

1 Obtained jointly by R. Sharpley. 
(i) $\|T(f, g)\|_{1} \leqslant\|f\|_{1}\|g\|_{1}$,

(ii) $\|T(f, g)\|_{\infty} \leqslant\|f\|\|g\|_{\infty}$;

then

$$
T(f, g)^{* *}(t) \leqslant \int_{0}^{\infty} f^{* *}(t / s) g^{*}(s) \frac{d s}{s} .
$$

THEOREM 4. (i) Assume that condition (1) is verified, and there exists $\theta$ $>0$ such that $A^{-1}(t) B^{-1}(t) \leqslant \theta t C^{-1}(t)$; then

$$
\Lambda\left(\phi_{X}, A\right) \hat{\otimes}_{\pi} \Lambda^{*}\left(\phi_{Y}, B\right) \subseteq \Lambda\left(\phi_{Z}, C\right) .
$$

(ii) Let $A(t)=B(t)=C(t)=t^{\alpha}$, and assume that condition (1) is verified. Then

$$
\Lambda_{\alpha}\left(\phi_{X}\right) \hat{\otimes}_{\pi} \Lambda_{\alpha}\left(\phi_{Y}\right) \subseteq \Lambda_{\alpha}\left(\phi_{Z}\right)
$$

whenever $\phi_{Z}{ }^{\circ} t^{\alpha} / t \downarrow$.

The problem of giving a "nice" set of necessary and sufficient conditions for arbitrary r.i. spaces remains open. An example of the difficulties encountered is given by

THEOREM 5. A necessary and sufficient condition for $M(X) \hat{\otimes}_{\pi} M(Y) \subseteq$ $M(Z)$ is that $\exists \theta>0$ such that

$$
\int_{0}^{\infty} \frac{1}{\phi_{X}(t / s)} \frac{1}{\phi_{Y}(s)} \frac{d s}{s} \leqslant \theta / \phi_{Z}(t) \quad \forall t>0,
$$

where $M(X)=\left\{f: \sup _{t>0}\left\{f^{* *}(t) \phi_{X}(t)\right\}<\infty\right\}$.

4. We consider the projective tensor product of r.i. spaces as $L^{1}$ (resp. $L^{\infty}$ ) modules (cf. [2], [9]).

THEOREM 6. (i) A necessary condition for $X \hat{\otimes}_{L^{1}} Y \subseteq Z$ is that $\exists \theta>0$ such that

$$
\phi_{Z}(t) t \leqslant \theta \phi_{X}(t) \phi_{Y}(t) \quad \forall t>0 .
$$

(ii) A necessary condition for $X \dot{\otimes}_{L^{\infty}} Y \subseteq Z$ is that $\exists \theta>0$ such that

$$
\phi_{Z}(t) \leqslant \theta \phi_{X}(t) \phi_{Y}(t) \quad \forall t>0 .
$$

THEOREM 7. (i) Assume that $\phi_{X}, \phi_{Y}, \phi_{Z}$ are bounded away by powers from 1 and $t$, that $\exists p_{i}$ such that $A_{i}(t) / t^{p_{i}} \downarrow$, that condition (3) is verified and, furthermore, that $\exists \theta>0$ such that $A_{1}^{-1}(t) A_{2}^{-1}(t) \leqslant \theta A_{3}^{-1}(t) \forall t>0$; then

$$
\Lambda\left(\phi_{X}, A_{1}\right) \grave{\otimes}_{L^{\infty}} \Lambda\left(\phi_{Y}, A_{2}\right) \subseteq \Lambda\left(\phi_{Z}, A_{3}\right) .
$$

(ii) If we assume that all the conditions on (i) are verified and replace condition (3) by condition (2) we obtain 


$$
\Lambda\left(\phi_{X}, A_{1}\right) \hat{\otimes}_{L}{ }_{L} \Lambda\left(\phi_{Y}, A_{2}\right) \subseteq \Lambda\left(\phi_{Z}, A_{3}\right)
$$

These results can be easily extended to Hardy classes $H\left(\phi_{X}, C\right)$ and $L_{\phi_{X}, C, \lambda}$ spaces (cf. [3], [8]). Detailed proofs and applications will be given elsewhere.

\section{REFERENCES}

1. A. P. Calderón, Intermediate spaces and interpolation, the complex method, Studia Math. 24 (1964), 113-190. MR 29 \#5097.

2. T. Donaldson, Harmonic analysis for functors on categories of Banach spaces of distributions, Trans. Amer. Math. Soc. 185 (1973), $1-82$.

3. P. Krée, Interpolation d'espaces vectoriels qui ne sont ni normés, ni complets applications, Ann. Inst. Fourier (Grenoble) 17 (1967), fasc. 2, 137-174 (1968). MR 37 \#4605.

4. W.A.J. Luxemburg, Rearrangement-invariant Banach function spaces, Queen's Papers in Pure and Appl. Math., no. 10, Queen's University, Kingston, Ont., 1967, pp. 83-144.

5. M. Milman, Integral transforms of weak type between rearrangement invariant spaces, Bull Amer. Math. Soc. 81 (1975), 761-762.

6. - Interpolation of bilinear operations on rearrangement-invariant spaces, Research Report, Australian National Univ., 1975.

7. R. O'Neil, Integral transforms and tensor products on Orlicz spaces and $L(p, q)$ spaces, J. Analyse Math. 21 (1968), 1-276.

8. J. Peetre, On the theory of $L_{p, \lambda}$ spaces, J. Functional Analysis 4 (1969), $71-87$. MR $39 \# 3300$

9. M. A. Rieffel, Multipliers and tensor products of $L^{p_{\text {-spaces }} \text { of locally compact }}$ groups, Studia Math. 3 (1969), 71-82. MR 39 \#6078.

10. A. Torchinsky, Interpolation of operations and Orlicz classes (preprint).

DEPARTMENT OF PURE MATHEMATICS, SCHOOL OF GENERAL STUDIES, AUSTRALIAN NATIONAL UNIVERSITY, AUSTRALIA A.C.T. 2600 